\title{
Efectividad de la terapia vocal versus tiroplastía en la voz de mujeres transgénero: una revisión integrativa
}

\section{Effectiveness of vocal therapy versus thyroplasty in the voice of transgender women: an integrative review}

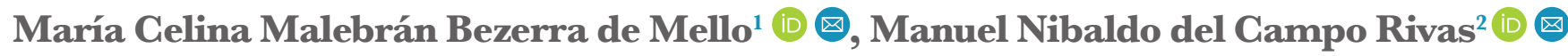 \\ ${ }^{1}$ Escuela de Fonoaudiología; Facultad de Ciencias; Universidad Mayor; Chile. \\ ${ }^{2}$ Escuela de Fonoaudiología; Facultad de Salud; Universidad Santo Tomás; Chile.
}

\section{Correspondencia}

María Celina Malebrán Bezerra de Mello, email: maria.malebran@umayor.cl

\section{Gitar así}

Malebrán Bezerra de Mello, María Celina; del Campo Rivas, Manuel Nibaldo. (2021). Efectividad de la terapia vocal versus tiroplastía en la voz de mujeres transgénero: una revisión integrativa. Revista de Investigación e Innovación en Ciencias de la Salud. 3(1): 48-60. https://doi. org/10.46634/riics.53

Recibido: $18 / 01 / 2021$

Revisado: $23 / 03 / 2021$

Aceptado: 23/06/2021

Editora invitada

Lady Catherine Cantor-Cutiva, Ph.D. (DD

Editor in chief

Jorge Mauricio Cuartas Arias, Ph.D. (iD)

Coeditor

Fraidy-Alonso Alzate-Pamplona, MSc. (iD

Gopyright $($ 2021. Fundación Universitaria María Cano. La Revista de Investigación e Innovación en Ciencias de la Salud proporciona acceso abierto a todo su contenido bajo los términos de la licencia Creative Commons Attribution-NonCommercial-NoDerivatives 4.0 International (CG BY-NG-ND 4.0).

Declaración de intereses

Los autores han declarado que no hay conflicto de intereses.

Disponibilidad de datos

Todos los datos relevantes se encuentran en el artículo. Para mayor información, comunicarse con el autor de correspondencia.

\section{Resumen}

Introducción. Las mujeres transgénero encuentran frecuentemente problemas en ajustar su voz a la frecuencia femenina. Para favorecer el cambio vocal deseado por estos individuos, se ofrecen dos técnicas: una quirúrgica (tiroplastía) y otra clínica (terapia vocal).

Objetivo. Esta revisión integrativa pretende verificar las publicaciones científicas relacionadas con las dos técnicas, observando cuál reporta mayor efectividad.

Método. Se realizó una búsqueda en bases de datos, considerando las palabras clave: Transsexual Persons AND Voice Feminization AND Thyroplasty OR Voice Training.

Resultados. Nueve artículos corresponden a estudios observacionales y tres utilizaron una metodología cuasi experimental. Los grupos estudiados incluyen un amplio espectro etario. Ninguno de los estudios informa aspectos económicos de las intervenciones. No existe evidencia de seguimiento a largo plazo.

Conclusiones. La vía de tratamiento vocal con adición hormonal puede ser una primera línea, y en caso de no reportar mejoras, la cirugía aparece como otra opción.

Recomendación. La recomendación es que se realice terapia vocal, que no implica riesgos significativos de complicaciones, siendo además un abordaje relativamente económico.

\section{Palabras clave}

Transgéneros; calidad vocal; entrenamiento vocal; laringoplastía; tiroplastía; vocología; fonoaudiología; voz.

\section{Abstract}

Introduction. Transsexual women frequently encounter problems in adjusting their voices to the characteristics of their new gender. In order to help in the change to the desired voice, two techniques are often used: the surgical (Thyroplasty) and clinical (Speech Therapy). 
Financiamiento

Ninguno. Esta investigación no recibió ninguna subvención específica de agencias de financiamiento en los sectores público, comercial o sin fines de lucro.

\section{Descargo de responsabilidad} El contenido de este artículo es responsabilidad exclusiva de los autores y no representa una opinión oficial de sus instituciones ni de la Revista de Investigación e Innovación en Ciencias de la Salud.
Objective. The aim of this integrative study is to verify through scientific publications the relationship between the two techniques and observe which is the most effective.

Method. A systematic database search was conducted considering the keywords: Transsexual Persons AND Voice Feminization AND Thyroplasty OR Voice Training.

Results. Nine articles corresponded to observational studies and three used a quasi-experimental methodology. The groups studied include a broad age spectrum and the quality of the evidence is low. No study reports economic aspects of the interventions. There is no evidence of long-term follow-up.

Conclusions. The vocal treatment route with hormonal addition can be a first line, and, in case of not reporting improvements, surgery appears as another option.

Recommendation. The recommendation is for Speech Therapy, which has no significant risks of complications and is relatively cheap.

\section{Keywords}

Transgender; voice quality; voice training; laryngoplasty; thyroplasty; vocology; Speech Language and Hearing Sciences; voice.

\section{Introducción}

La disforia de género fue considerada una condición de salud mental por el Manual Diagnóstico y Estadístico de los Trastornos Mentales (DSM-V) [1]. Para el diagnóstico, se sugería investigar al menos dos de las siguientes características que deben presentarse por un tiempo mínimo de 6 meses: marcada incongruencia entre el sexo que el individuo siente o expresa y sus caracteres sexuales primarios o secundarios; un fuerte deseo por desprenderse de los caracteres sexuales propios primarios o secundarios; un potente anhelo por poseer los caracteres sexuales (primarios y/o secundarios) correspondientes al sexo opuesto; pretensión de ser del otro sexo (o de un sexo alternativo distinto del que se le asigna); ser tratado como del otro sexo (o de un sexo alternativo distinto del que se le asigna); una fuerte convicción de tener los sentimientos y reacciones típicos del otro sexo (o de un sexo alternativo distinto del que se le asigna); descontento o depresión constante que perjudica aspectos sociales, escolares y/u otras importantes áreas de funcionamiento [1,2].

Sin embargo, la CIE-11 define al transexual no como una persona que padece una patología, sino como una condición en la cual aquel que presenta una incongruencia marcada y persistente entre el género experimentado del individuo y el sexo asignado, vinculado al deseo de transición para vivir y ser aceptado como una persona del género experimentado, a través del tratamiento hormonal, la cirugía u otras prestaciones sanitarias. Se sugiere que el abordaje no se dé antes del inicio de la pubertad [3].

Así, para ofrecer una adecuada transición al transexual, se debe conocer el programa médico ofrecido a estos sujetos [4]. Bajo este concepto, se entiende que una transición de género completa incluye un abordaje multi e interdisciplinario, con diálogo profesional constante y atenciones específicas en urología/ginecología, otorrinolaringología, endocrinología, cirugía plástica, psicología, fonoaudiología, entre otros. 
La voz es considerada la extensión de la personalidad, y los transexuales femeninos (TF) vivencian un gran desafío: sus voces y características de la comunicación generalmente contrastan con el género deseado al final del proceso de reasignación sexual. Esta discrepancia termina por exponer el género biológico original del individuo, frecuentemente resultando en una dificultad en su aceptación social $[4,5]$.

Para los transexuales masculinos, los tratamientos hormonales poseen un efecto en la laringe que agrava la frecuencia (pitch) a una extensión masculina. Por lo mismo, este grupo buscará menos los servicios de terapia vocal. Sin embargo, para los TF, el tratamiento hormonal no afectará al pitch, por lo que la terapia vocal es una opción para desarrollar una voz femenina socialmente aceptable [6]. Parece lógico que el fonoaudiólogo especialista en voz sea el profesional idóneo para conducir la terapia vocal de los TF, quienes, por definición, no se sienten representados por su imagen corporal y vocal, experimentando una fragmentación en la extensión de sus personalidades [4].

La literatura menciona dos dimensiones relevantes a considerar para la terapia vocal de transexuales: aspectos comunicacionales basados en la estructura genética/biológica (características sexuales primarias y/o secundarias) y aspectos basados en comportamientos sociales aprendidos, como la prosodia, los patrones articulatorios, la selección de palabras, la comunicación gestual, los códigos de vestimenta, entre otros [7]. Una investigación señala que, tras terapia vocal a transexuales masculinos sometidos a terapia con andrógenos de larga data que abarcaba determinación del pitch, prosodia y parámetros de perturbación medidos en tareas vocales específicas, la mayoría de los sujetos presentaba una voz masculina aceptable, pero $10 \%$ de ellos presentaron alguna dificultad que podría relacionarse a una sensibilidad disminuida al andrógeno [8].

La terapia vocal en TF se enfoca tradicionalmente en el aumento del pitch o del promedio de la frecuencia fundamental (f0) de la voz [9-12]. Ahora, la agudización no debe ser la única propuesta terapéutica; hay que recordar que tanto hombres como mujeres poseen superposición de frecuencias que permiten una prosodia variada y flexible a diferentes situaciones del día a día.

Una investigación concluyó que el promedio de las f0 en el habla de hombres con edades entre 20 y 69 años variaba entre 107 y $132 \mathrm{~Hz}$, mientras que el promedio para las mujeres en el mismo rango etario variaba entre 189 y $224 \mathrm{~Hz}$ [5]. En relación a la intensidad utilizada en el habla, se advierte el uso ligeramente más elevado en la voz de hombres jóvenes (70.42 dB), comparada a la de jóvenes mujeres $(68.15 \mathrm{~dB})$ [13]. Es común pensar que la f0 deba ser elevada, incluso en falsete - un abordaje en desuso por la dificultad de mantención de esta voz y la poca autenticidad que entrega [4]. Otras técnicas vocales también han sido mencionadas en artículos científicos para abordar la terapia vocal a pacientes transexuales, como la terapia de resonancia oral y los ejercicios para la función vocal de Stemple (Vocal Function Exercises, VFE), aunque este último sin resultados óptimos [6,14].

A parte de la f0 y la intensidad, se ha reportado que el entrenamiento auditivo y la práctica gradual producen leve ascenso de la frecuencia fundamental [4]. Otros autores sugieren enfocar la terapia de feminización vocal en técnicas articulatorias. Sin embargo, indican que se deben hacer más investigaciones para reforzar la evidencia y establecer así si existe realmente una contribución de la resonancia oral y cambios de la f0 en la percepción de la feminización vocal [14]. 
También se sugiere mejorar el soporte respiratorio y la eficiencia de la válvula laríngea para la mantención de una calidad vocal más suave, mientras los ajustes vocales en el pitch son trabajados en la terapia vocal. Así, se enfatizaría la resistencia, equilibrio y coordinación de la respiración, fonación y sistemas resonanciales [6].

Respecto a la duración de los efectos logrados posterior a la terapia vocal, un estudio evaluó la percepción auditiva de voces de transexuales femeninas sometidas a fonoterapia en tres situaciones: previo a terapia vocal, inmediatamente tras recibir terapia vocal y en un seguimiento de control de alta. Los autores refieren cambios acústicos significativos en las dos últimas situaciones (tras recibir terapia vocal y en control post alta) y sugieren que 8 semanas de terapia vocal pueden resultar en cambios vocales en sujetos TF, permaneciendo los beneficios de la terapia por al menos 15 meses [15].

Además de la oferta de terapia vocal para cambio de f0 y características vocales - y con esto mayor acercamiento a la veracidad del género de interés - las mujeres transgénero también pueden recurrir a la cirugía laríngea para elevación del pitch.

Una revisión previa de la efectividad de la cirugía de elevación del pitch, realizada a individuos TF, identificó un aumento significativo de la f0 en el postoperatorio. Perceptivamente, la formación del web laríngeo pareció riesgosa para una peor calidad vocal. La gran mayoría de los pacientes demostró satisfacción con su nueva voz postcirugia. Sin embargo, ninguno de los estudios usó un grupo control ni procesos aleatorios. Se necesitan más investigaciones que indiquen los resultados a largo plazo. Los autores también sugieren que investigaciones futuras deben investigar los efectos a largo plazo de la cirugía de aumento de la frecuencia, mediante un diseño de estudio más sólido [16].

La literatura refiere que una vez que el sujeto está viviendo con el género reasignado y la terapia vocal ha concluido, y aun así se advierte el deseo de mejorar el pitch, las modificaciones quirúrgicas pueden ser una alternativa razonable. Sin embargo, se observa que la cirugía laríngea no es definitiva. Como ejemplo, en la técnica de Isshiki (tiroplastía tipo IV), el resultado quirúrgico de feminización vocal no se mantiene en el tiempo, y el pitch tiende a desplazarse a los graves. La técnica quirúrgica que produce mejores efectos a largo plazo es, según los autores, la de fusión cricotiroidea. Entretanto, esta técnica es ofrecida solo a transexuales jóvenes, ya que necesita cierta flexibilidad del cartílago cricoides para su ubicación dentro del borde inferior del cartílago tiroideo [17]. El objetivo de la cirugía de cuerdas vocales es reducir su extensión vibratoria, elevando la frecuencia fundamental y reduciendo la ronquera asociada [4].

Diversos autores concuerdan en que la técnica quirúrgica conocida por glotoplastía de Wendler contribuye en la feminización vocal. Los pacientes que se sometieron a esta cirugía, seguida de terapia vocal, experimentaron un aumento significativo de la frecuencia fundamental, pero también un aumento en el grado de la disfonía y de la presión subglótica estimada. Un estudio evaluó a 31 sujetos transgénero que pasaron por cirugía laríngea con la misma técnica de Wendler, seguida de terapia vocal, observando por 9,2 meses a dos grupos: uno con sujetos menores a 40 años y otro con sujetos mayores a esa edad. Ambos grupos experimentaron un aumento de la f0, especialmente el grupo con sujetos más jóvenes. La frecuencia promedio tuvo una tendencia a decrecer en el postoperatorio, al mismo tiempo en que la presión subglótoica esperada aumentó considerablemente. Como conclusión, los autores comentaron que la técnica es efectiva, con efectividad aún mayor en individuos más 
jóvenes $[17,18]$. En otro estudio, se refuerza la idea que la técnica de Wendler modificada por Hagen es un método efectivo y de bajo riesgo en la elevación de la f0 de transgéneros. Sin embargo, puntajes elevados en el Índice de Discapacidad Vocal (Voice Handicap Index - VHI) indicaron que en actividades diarias los transgéneros continuaban sintiéndose deficientes debido a sus voces, llevando a una falta de aceptación e integración social, cuestión que también se investigó en este estudio por medio de la encuesta Life Satisfaction Questionnaire, razón por la cual los autores refuerzan la necesidad de un concepto de terapia de feminización vocal multidisciplinaria [19].

En un estudio de cohorte retrospectivo, el autor relata los resultados vocales de un grupo de 10 personas transgénero sometidas a una cirugía para reducir la longitud de los pliegues vocales. Se analizaron aspectos demográficos, evaluación con videoestroboscopía y datos acústicos pre y postoperatorios. En promedio, se constató un aumento de $110 \mathrm{~Hz}$ en la fo luego de la cirugía. Su estudio concluye comentando que esta nueva técnica vía endoscópica ha demostrado ser un procedimiento exitoso para la elevación permanente de la f0 con poca o ninguna morbilidad [20].

Orloff (2006) y Geneid (2015) mencionan otras técnicas como LAVA (ajuste vocal por medio de asistencia con láser). Ambos autores comentan el éxito de sus técnicas quirúrgicas en el aumento de la f0 $[21,22]$.

El objetivo de esta revisión fue conocer el estado del arte en relación a la efectividad de la terapia vocal versus la tiroplastía en mejeres transgénero.

\section{Metodología}

Se realizó una revisión integrativa [23] de la literatura a partir de la siguiente pregunta de investigación: ¿cuál es la efectividad clínica de la terapia vocal al ser comparada con tiroplastía en mujeres transgénero, considerando como desenlace principal la elevación de la f0? La búsqueda se ejecutó en las bases de datos Oxford Journals, ISI Web of Science, Science Direct, Springer Link, Medline Complete, Psychology and Behavioral Sciences Collection y Pubmed.

Las palabras clave para ejecutar la búsqueda fueron obtenidas del Medical Subject Headings (MeSH), combinadas con los booleanos AND/OR. A saber, Transsexual Persons AND Voice Feminization AND Thyroplasty OR Voice Training.

Las restricciones para escoger las publicaciones fueron artículos cuyo acceso fuera completo y gratuito en la red, con fechas de publicación entre los años 2006-2021 y que estuvieran escritos en idioma inglés. La búsqueda se realizó en dos instancias, inicialmente entre los días 02-09-2016 y 05-09-2016, y posteriormente entre 07-05-2021 y 22-05-2021, con el fin de actualizar la información disponible.

Para el análisis de los datos se solicitó a dos fonoaudiólogos independientes revisar la pertinencia de los artículos en consideración del tema central del estudio, eliminando de la selección: a) cualquier artículo distinto a una investigación original, b) estudios cuya orientación no se centrara en el grupo de interés y c) reportes que no representaran una aproximación al tratamiento de feminización vocal.

El flujograma de artículos identificados, seleccionados y analizados se representó a partir de la declaración PRISMA [24]. Posteriormente se procedió a ejecutar la revisión íntegra de resúmenes y textos completos, analizando metodología de los estudios, población objetivo, 
tipo de tratamiento, desenlaces clínicos (cambios en fo, feminización vocal, disfonía), autosatisfacción vocal y complicaciones. A partir de dicha exploración se agrupó la información comparable de los artículos en una tabla de evidencia y se realizó tanto la descripción como el análisis crítico de los textos.

\section{Resultados}

Se identificaron 309 artículos en las bases de datos. La eliminación de duplicados permitió contar con una matriz de 297 artículos, de los que se eliminaron 281 que no cumplieron con los criterios de selección, de acuerdo con la información otorgada por su título o resumen, quedando finalmente 11 textos, que fueron analizados de forma completa (ver Figura 1 y Tabla 1).

La mayor parte de los artículos $(73 \%)$ corresponden a estudios observacionales $[6,15,17,18,20,22,25,26]$ y solo tres de ellos $(27 \%)$ utilizaron una metodología cuasi experimental $[14,19,21]$. En relación con la temporalidad de los estudios, cinco $(45 \%)$ fueron retrospectivos $[17,18,20,26,22]$ y seis $(55 \%)$ longitudinales prospectivos $[6,14,15,19,21,25]$.

En el periodo 2013 a 2016 se advirtió un incremento considerable en la publicación de artículos originales vinculados a la pregunta de investigación, contando con ocho $(73 \%)$ de los 11 estudios seleccionados en la presente revisión [6,15,18,19,20,22,25,26]. Entre 2017 a 2021 se detectó la publicación de revisiones o adaptaciones de instrumentos para la evaluación de aspectos funcionales asociados a la voz y calidad de vida de la mujer transgénero, no así experiencias de intervención en las bases de datos exploradas.

Todas las experiencias de intervención elegidas involucraron en su abordaje a personas mayores de 18 años, reportando heterogeneidad en la selección y conformación de los grupos estudiados, con participación de usuarias con edades hasta los 65 años en algunos casos. Ninguno de los estudios seleccionados utilizó una metodología experimental con diseño de ensayo clínico controlado aleatorizado (ECA).

Desde el punto de vista de los tipos de intervención, estos fueron variados y en ocasiones simultáneos, entendiendo que ciertos grupos de pacientes recibieron tratamiento vocal por parte de fonoaudiólogos $[6,14,15]$, laringoplastía [17-19,21,26], tratamiento combinado vocal y quirúrgico [22,25] o vocal, quirúrgico y prescripción hormonal [20].

Respecto a la periocidad, dosis y tiempo de seguimiento, se aprecia de igual forma una amplia gama de diseños, cuyos resultados, en general, reportan beneficios y pocas complicaciones para la feminización vocal de las personas transgénero, siendo aparentemente el más efectivo (incremento f0 $110 \mathrm{~Hz}$; $\mathrm{p}=0.001$ ), aquel que reúne terapia vocal, terapia de reemplazo hormonal y cirugía laríngea, en caso de requerirlo [20], de manera racional y de acuerdo a las necesidades de la usuaria.

Uno de los resultados principales tratados por los artículos [14,17-22,25,26] es la modificación de la fo en la voz de la mujer transgénero como resultado del tratamiento. De igual forma, se consideró la percepción de feminización vocal $[6,14,15,18,19,21,25,26]$ y la autosatisfacción vocal $[17,19,21,25,26]$, como aspectos a evaluar de forma transversal y con resultados reportados positivos.

Cuatro artículos refirieron complicaciones postratamiento, vinculadas a grados variables de disfonía [17,18,20,21], al desarrollo de alteraciones inflamatorias como granuloma [20,22] y a la presencia de complicaciones que obligaron realizar revisiones quirúrgicas $[18,19]$ en el caso de tiroplastías. 


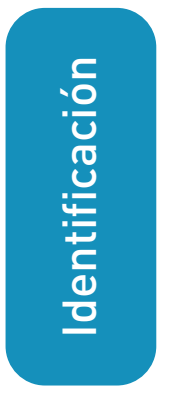

Identificación de artículos a través de la búsqueda en 7 bases de datos (n = 309)
Artículos adicionales identificados a través de otras fuentes $(n=0)$

$\downarrow$
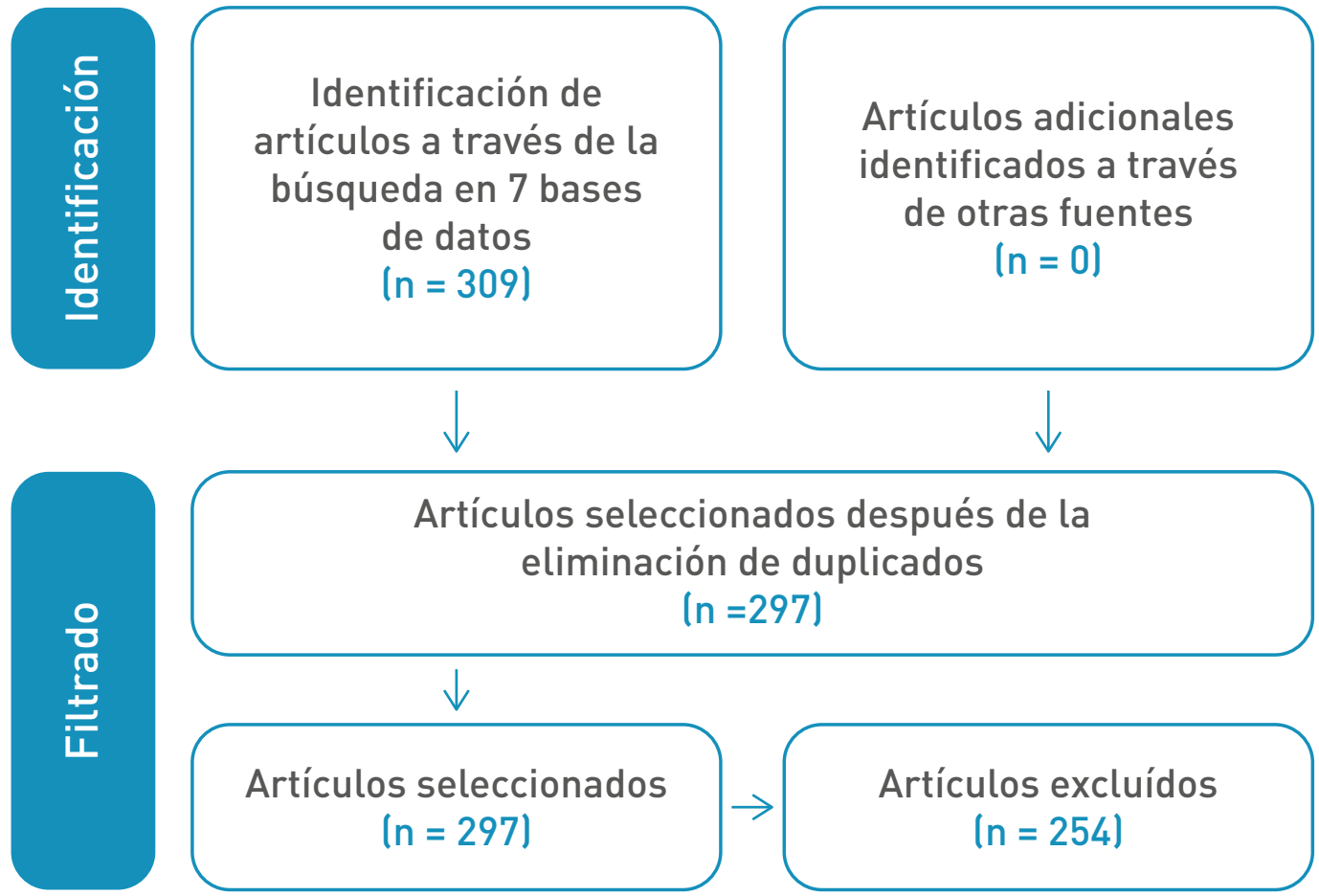
eliminación de duplicados

$$
\text { ( } n=297 \text { ) }
$$

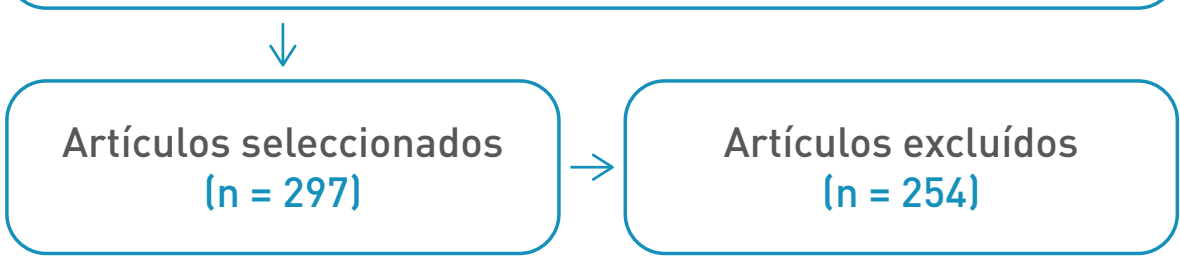

Artículos completos evaluados dependiendo de su elegibilidad ( $n=43$ )

Artículos completos eliminados por razones particulares

$$
\text { ( } n=32 \text { ) }
$$
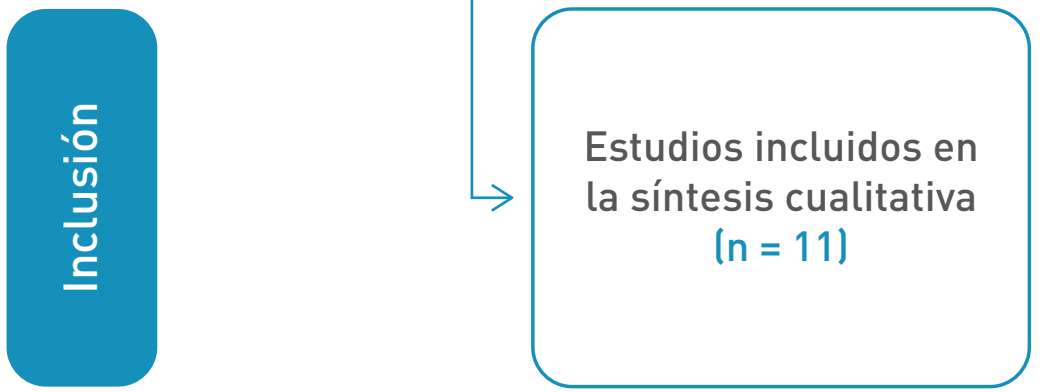

Figura 1. Flujograma de la selección de estudios 


\section{Tabla 1. Matriz de evidencias aplicada a la revisión}

\begin{tabular}{|c|c|c|c|c|c|c|c|c|}
\hline \multicolumn{4}{|c|}{ Tratamiento } & \multicolumn{5}{|c|}{ Resultados } \\
\hline $\begin{array}{l}\text { Autor/ } \\
\text { año }\end{array}$ & Vocal & Hormonal & Quirúrgico & fo & Feminización & Disfonía & $\begin{array}{l}\text { Autosatis- } \\
\text { facción vocal }\end{array}$ & Complicaciones \\
\hline $\begin{array}{l}\text { Remacle } \\
\text { et al. } \\
\text { (2011) }\end{array}$ & & & $\begin{array}{l}\text { Glotoplastía- } \\
\text { técnica } \\
\text { Wendler con } \\
\text { modificaciones } \\
\text { menores }\end{array}$ & $\begin{array}{l}\text { Incremento } \\
\text { de } 52 \mathrm{~Hz} \\
(p=0,006)\end{array}$ & & $\begin{array}{c}\text { Aumento } \\
\text { en el grado } \\
\text { de disfonía } \\
\text { (Gpre=0.2, G } \\
\text { post }=1 . \\
P=0.013)\end{array}$ & $\begin{array}{c}\text { Mayor post } \\
\text { tratamiento } \\
(p=0,047)\end{array}$ & \\
\hline $\begin{array}{l}\text { Carew et } \\
\text { al. (2007) }\end{array}$ & $\begin{array}{c}\text { Resonancia } \\
\text { oral }\end{array}$ & & & $\begin{array}{l}\text { Incremento } \\
(P=0.013)\end{array}$ & $\begin{array}{c}\text { Evidencia } \\
\text { preliminar de } \\
\text { la efectividad }\end{array}$ & & & \\
\hline $\begin{array}{l}\text { Gelfer et } \\
\text { al. }(2013\end{array}$ & $\begin{array}{c}\text { Tratamiento } \\
\text { sintomático } \\
\text { de la } \\
\text { voz más } \\
\text { ejercicios } \\
\text { funcionales } \\
\text { de Stemple }\end{array}$ & & & & $\begin{array}{l}\text { Voz más } \\
\text { similar a la } \\
\text { de controles } \\
\text { femeninos } \\
\text { post } \\
\text { tratamiento }\end{array}$ & & & \\
\hline $\begin{array}{l}\text { Gelfer et } \\
\text { al. (2013) }\end{array}$ & $\begin{array}{l}\text { Terapia } \\
\text { vocal de } \\
\text { grupo }\end{array}$ & & & & $\begin{array}{c}\text { Post terapia } \\
50,8 \% \text { de los } \\
\text { pacientes y a } \\
\text { los } 15 \text { meses, } \\
33,1 \% \text { de los } \\
\text { mismos }\end{array}$ & & & \\
\hline $\begin{array}{l}\text { Mastroni- } \\
\text { kolis. } \\
\text { (2013) }\end{array}$ & & & $\begin{array}{l}\text { Glotoplastía- } \\
\text { técnica } \\
\text { Wendler. } 3 \\
\text { casos habían } \\
\text { sido sometidos } \\
\text { previamente a } \\
\text { aproximación } \\
\text { cricotiroidea } \\
\text { en otro centro }\end{array}$ & $\begin{array}{c}\text { Incremento } \\
\text { de } 70,5 \mathrm{~Hz} \\
(p=0,001)\end{array}$ & $\begin{array}{l}\text { Tendencia } \\
\text { post cirugía }\end{array}$ & $\begin{array}{c}\text { El grado } \\
\text { de disfonía } \\
\text { aumentó } \\
\text { significativa- } \\
\text { mente post } \\
\text { operación, } \\
\text { de manera } \\
\text { especial en } \\
\text { uno de los } \\
\text { grupos -B- } \\
(p=0,035)\end{array}$ & & $\begin{array}{l}3 \text { pacientes } \\
\text { necesitaron } \\
\text { revisión de } \\
\text { la cirugía (no } \\
\text { cumplir con } \\
\text { indicaciones, } \\
\text { etc.). }\end{array}$ \\
\hline $\begin{array}{c}\text { Anderson. } \\
\text { (2013) }\end{array}$ & $\begin{array}{c}\text { Tratamiento } \\
\text { conductual } \\
\text { en inicio, } \\
\text { si no logra } \\
\text { confort } \\
\text { vocal se } \\
\text { ofreció } \\
\text { la opción } \\
\text { quirúrgica }\end{array}$ & $\begin{array}{l}\text { Terapia de } \\
\text { reemplazo } \\
\text { hormonal } \\
\text { (promedio } \\
\text { de } 7,2 \\
\text { años) }\end{array}$ & $\begin{array}{c}\text { Formación } \\
\text { de red glótica } \\
\text { anterior } \\
\text { asistida con } \\
\text { inyección de } \\
\text { aumento. } 2 \\
\text { pacientes } \\
\text { con cirugía } \\
\text { laríngea previa } \\
\text { no exitosa }\end{array}$ & $\begin{array}{l}\text { Incremento } \\
\text { de } 110 \mathrm{~Hz} \\
(p=0,001)\end{array}$ & & $\begin{array}{l}\text { Disfonía } \\
\text { moderada a } \\
\text { severa por } \\
\text { laringitis } \\
\text { post } \\
\text { quirúrgica (6 } \\
\text { semanas) }\end{array}$ & & $\begin{array}{l}1 \text { paciente } \\
\text { desarrolla } \\
\text { granuloma }\end{array}$ \\
\hline
\end{tabular}




\begin{tabular}{|c|c|c|c|c|c|c|c|}
\hline $\begin{array}{l}\text { Meister et } \\
\text { al. (2016) }\end{array}$ & & $\begin{array}{c}\text { Glotoplastía } \\
\text { de Wendler } \\
\text { modificada por } \\
\text { Hagen }\end{array}$ & $\begin{array}{l}\text { Incremento } \\
\text { a rangos } \\
\text { femeninos }\end{array}$ & $\begin{array}{l}\text { Elevación } \\
\text { del límite de } \\
\text { frecuencia } \\
\text { inferior, sin } \\
\text { pérdida del } \\
\text { rango de } \\
\text { frecuencias }\end{array}$ & $\begin{array}{c}\text { El índice de } \\
\text { gravedad de } \\
\text { la disfonía } \\
\text { no presentó } \\
\text { cambios }\end{array}$ & $\begin{array}{l}\text { Se reporta } \\
\text { una elevada } \\
\text { correlación } \\
\text { entre la } \\
\text { satisfacción } \\
\text { subjetiva de } \\
\text { la voz y la } \\
\text { elevación } \\
\text { de la Fo }\end{array}$ & $\begin{array}{l}1 \text { paciente } \\
\text { requiere } \\
\text { revisión de la } \\
\text { cirugía y } 1 / 3 \text { se } \\
\text { siente afectado } \\
\text { por el rango } \\
\text { dinámico de la } \\
\text { voz limitado. }\end{array}$ \\
\hline $\begin{array}{l}\text { Casado et } \\
\text { al. (2016) }\end{array}$ & $\begin{array}{c}\text { Terapia } \\
\text { vocal }\end{array}$ & Glotoplastía & $\begin{array}{l}\text { Incremento } \\
\text { de } 106 \\
\mathrm{~Hz} .(p=0.005)\end{array}$ & $\begin{array}{c}\text { Mejora en } \\
\text { el grado de } \\
\text { feminización } \\
\text { vocal ( } p=0,04 . \\
\text { SD: } 0.57)\end{array}$ & & $\begin{array}{l}\text { Cambio } \\
\text { en la } \\
\text { autosatis- } \\
\text { facción } \\
\text { personal } \\
\text { de la voz } \\
(p=0.05)\end{array}$ & \\
\hline $\begin{array}{c}\text { Thomas } \\
\text { et al. } \\
(2013)\end{array}$ & & $\begin{array}{c}\text { Laringoplastía } \\
\text { de } \\
\text { feminización } \\
\text { (modificación } \\
\text { del cartílago } \\
\text { tiroides } 22 \\
\text { pacientes) + } \\
\text { Aproximación } \\
\text { tirohioidea (54 } \\
\text { personas) }\end{array}$ & $\begin{array}{c}\text { Incremento } \\
\text { de } 57 \mathrm{~Hz} \\
(p=0.01)\end{array}$ & $\begin{array}{c}\text { Si la cirugía } \\
\text { es exitosa, } \\
\text { elimina las } \\
\text { notas bajas } \\
\text { del rango } \\
\text { vocal. }\end{array}$ & & $\begin{array}{l}\text { Incrementa } \\
\text { el confort } \\
\text { respecto a } \\
\text { la Fo }\end{array}$ & \\
\hline $\begin{array}{l}\text { Geneid et } \\
\text { al. (2015) }\end{array}$ & $\begin{array}{l}\text { Terapia } \\
\text { vocal y } \\
\text { cirugía } \\
\text { como } \\
\text { opción } \\
\text { frente a } \\
\text { la falta de } \\
\text { resultados }\end{array}$ & $\begin{array}{c}\text { Cirugía } \\
\text { plástica en } \\
\text { capa mucosa } \\
\text { de la cuerda } \\
\text { vocal. Técnica } \\
\text { combinada } \\
\text { de sutura } \\
\text { anterior } \\
\text { transmucosa y } \\
\text { rigidización de } \\
\text { cuerda vocal } \\
\text { a través de } \\
\text { una incisión } \\
\text { de cordotomía } \\
\text { longitudina con } \\
\text { láser C02 }\end{array}$ & $\begin{array}{l}\text { Incremento } \\
\text { a largo } \\
\text { plazo } \\
(p=0.009)\end{array}$ & & & & $\begin{array}{l}1 \text { paciente } \\
\text { reporta } \\
\text { síntomas de } \\
\text { perforación } \\
\text { anterior y } \\
\text { granulación del } \\
\text { tejido }\end{array}$ \\
\hline $\begin{array}{l}\text { Orloff et } \\
\text { al. (2006) }\end{array}$ & & $\begin{array}{l}\text { Ajuste vocal } \\
\text { asistido por } \\
\text { cirugía láser } \\
\text { en hombre } \\
\text { a mujer con } \\
\text { androfonía }\end{array}$ & $\begin{array}{c}\text { Incremento } \\
\text { de } 26 \mathrm{~Hz} \text {. } \\
\text { (I.C: } 95 \% ; \\
\text { t-test } \\
\text { p<.0025) }\end{array}$ & $\begin{array}{c}6 / 10 \text { voces } \\
\text { de pacientes } \\
\text { fueron } \\
\text { percibidos } \\
\text { como } \\
\text { femeninas. }\end{array}$ & $\begin{array}{l}\text { Disminución } \\
\text { de la } \\
\text { calidad, } \\
\text { volumen y } \\
\text { rango vocal. }\end{array}$ & Incrementa & \\
\hline
\end{tabular}




\section{Discusión}

A razón del análisis de los artículos antes presentados, si bien se informan resultados estadísticamente significativos en algunos de ellos, los hallazgos finales se enmarcan en experiencias integrales de tratamiento. La selección de las usuarias, en general, incluyó a muestras pequeñas que no fueron representativas y se enmarcaban en el desarrollo de actividades terapéuticas de centros especializados en temas de la entonces denominada "disforia de género". Por lo tanto, es poco recomendable que los hallazgos sean extrapolados a la población general de mujeres transgénero (Tabla 1) [27].

Debe considerarse, en este sentido, que el estudio de la población transgénero es complejo, puesto que el proceso de transición se desarrolla en ámbitos de privacidad individual y/o familiar, lo que dificulta el acceso a muestras amplias de la población [28].

Teniendo en cuenta los resultados obtenidos en la revisión, la cirugía laríngea puede reportar beneficios que se reflejan en la calidad vocal de TF, con bajo riesgo de complicaciones. Sin embargo, se recomienda como primera línea el tratamiento vocal brindado por un fonoaudiólogo experto en voz, con el propósito de evitar someter a la persona a una situación estresante como la cirugía. Ambos tratamientos responden a tiempos distintos: la cirugía genera un cambio relativamente rápido, en comparación con la terapia vocal, que requiere de por lo menos entre 6 a 16 sesiones para reportar beneficios objetivos) [6,14,15]. Ahora bien, el tratamiento hormonal reporta de igual modo una modificación en aspectos objetivos de la voz.

No se registran estudios en el impacto vocal de adolescentes y niños [29], quienes comienzan su transición tempranamente con terapia de reemplazo hormonal y asistencia psicológica. Hay reportes de que la terapia hormonal, en transgéneros adultos y en conjunto con otras técnicas terapéuticas, colabora en el proceso de feminización vocal de manera efectiva [20]. Otro aspecto importante a considerar es que, a mediano plazo, se reporta la estabilidad en el cambio vocal asociado a la transición de género, aunque es posible que, a largo plazo, se recuperen segmentos del rango vocal propios del estadio previo a la reasignación [15].

Existe una dimensión psicológica que debe ser mencionada, en relación a la necesidad de olvidar todo aquello vinculado con el género antes de la reasignación de sexo [30], lo que puede interrumpir vías terapéuticas bien encaminadas, afectando finalmente en la calidad de vida. Esto se debe a que a largo plazo la percepción de la voz masculinizada o feminizada por parte de oyentes externos, en situaciones de la vida diaria como hablar por teléfono, pedir una cuenta, etc., exponen a la persona y ello reporta un menoscabo en la calidad de vida.

Contar con un especialista de la voz que asesore y acompañe el proceso colaboraría con la consolidación de una voz feminizada en el transgénero femenino. Sin embargo, se reitera que la terapia fonoaudiológica no se debe centrar solo en la agudización de la frecuencia fundamental. Trabajar otros aspectos que influyen en la calidad vocal es fundamental: ajustes supraglóticos, flexibilización de patrones articulatorios, control de la resonancia son puntos importantes a considerar. Los monitoreos propioceptivos y auditivos también deben ser reforzados, así como aspectos de habla y psicodinámica vocal. Finalmente, comprender que la voz es dinámica y muchas veces reflejo de aspectos socioculturales. Con todo, cabe destacar que no existe evidencia de seguimiento a largo plazo, con control de factores intervinientes que puedan asegurar la aseveración previa.

Si no se logran cambios o estos no son satisfactorios, se recomienda continuar hacia la cirugía laríngea, entregando esta indicación como una sugerencia débil. Debe tenerse en 
perspectiva la posibilidad de complicaciones, aunque su reporte es bajo [26]. La terapia de reemplazo hormonal aplicada de manera paralela y con supervisión médica reporta de igual modo cambios que coadyuvan a feminizar la voz. Cabe resaltar que es necesario realizar el tratamiento con supervisión médica, en dosis y frecuencia ajustadas por un especialista.

Considerando la evidencia antes detallada, es posible recomendar el uso de tratamiento vocal y/o cirugía laríngea para modificar la voz del transgénero. Sin embargo, la decisión debe ser tomada de manera individual y se requiere ofrecer más información, con el fin de tomar decisiones ajustadas a sus necesidades y a los resultados efectivos que la intervención, cualquiera que sea, pueda tener en la voz.

La presente revisión enfrenta diversas limitaciones. Primero, los artículos seleccionados sólo consideraron el idioma inglés, por lo que existe la posibilidad de no contar con experiencias informadas en otros idiomas. Segundo, las investigaciones examinadas no informaron aspectos económicos de las intervenciones, lo cual entorpece la determinación de recomendaciones que incluyan este aspecto. Tercero, los grupos estudiados incluyeron un amplio espectro etario. Finalmente, ninguno de los artículos siguió una metodología experimental, con aleatorización y cegamiento de intervenciones, por lo que la calidad global de la evidencia es baja.

\section{Conclusiones}

La evidencia antes recopilada no permite decidir a favor o en contra de un tratamiento en particular. Podemos concluir que el abordaje vocal con adición hormonal puede ser una primera línea y en caso de no reportar mejoras, la cirugía aparece como otra opción, no definitiva, que, en caso de ser respaldada por especialistas de la voz, colaboraría en el bienestar, la feminización y la autosatisfacción vocal de mujeres transgénero.

\section{Referencias}

1. American Psychiatric Association. Diagnostic and Statistical Manual of Mental Disorders [Internet]. Fifth Edition. Washington: American Psychiatric Association; 2013 [citado 17 de junio de 2021]. Doi: https://doi.org/10.1176/appi.books.9780890425596

2. Markwick L. Male, Female, Other: Transgender and the Impact in Primary Care. The Journal for Nurse Practitioners. Mayo de 2016;12(5):330-8. Doi: https://doi.org/10.1016/j.nurpra.2015.11.028

3. Amigo-Ventureira AM. Un recorrido por la historia trans*: desde el ámbito biomédico al movimiento activista-social. Cad Pagu. 2019;(57):e195701. Doi: https://doi.org/10.159 0/18094449201900570001

4. Freeman M, Fawcus M, editores. Voice disorders and their management. 3. ed., reprinted. London: Whurr; 2001.

5. Colton R, Casper J, Leonard R. Understanding voice problem: A physiological perspective for diagnosis and treatment. Differential Diagnosis of Voice Problems. 4th ed. Philadelphia: Wolters Kluwer Health Adis (ESP); 2011.

6. Gelfer MP, Van Dong BR. A Preliminary Study on the Use of Vocal Function Exercises to Improve Voice in Male-to-Female Transgender Clients. Journal of Voice. Mayo de 2013;27(3):321-34. Doi: https://doi.org/10.1016/j.jvoice.2012.07.008 
7. Sataloff R. Professional Voice: The Science and Art of Clinical Care. 3rd ed. San Diego: Plural Publishing Group; 2005.

8. Cosyns M, Van Borsel J, Wierckx K, Dedecker D, Van de Peer F, Daelman T, et al. Voice in female-to-male transsexual persons after long-term androgen therapy: Voice in Female-to-Male Transsexuals. The Laryngoscope. Junio de 2014;124(6):1409-14. Doi: https:// doi.org/10.1002/lary.24480

9. Dacakis G. Long-term maintenance of fundamental frequency increases in male-to-female transsexuals. Journal of Voice. Diciembre de 2000;14(4):549-56. Doi: https://doi. org/10.1016/S0892-1997(00)80010-7

10. Oates J, Dacakis G. Voice change in transsexuals. Venerology. 1997;10:178-87. Disponible en: https://n9.cl/z1k5b

11. Kalra MA. Voice Therapy with a Transsexual. In: Gemme R, Wheeler CG, editores. Progress in Sexology. Perspectives in Sexuality (Behavior, Research, and Therapy). Boston: Springer; 1997. Doi: https://doi.org/10.1007/978-1-4684-2448-5_10

12. Gelfer MP, Schofield KJ. Comparison of acoustic and perceptual measures of voice in male-to-female transsexuals perceived as female versus those perceived as male. Journal of Voice. Marzo de 2000;14(1):22-33. Doi: https://doi.org/10.1016/S08921997(00)80092-2

13. Gelfer MP, Young SR. Comparisons of intensity measures and their stability in male and female sneakers. Journal of Voice. Junio de 1997;11(2):178-86. Doi: https://doi. org/10.1016/S0892-1997(97)80076-8

14. Carew L, Dacakis G, Oates J. The Effectiveness of Oral Resonance Therapy on the Perception of Femininity of Voice in Male-to-Female Transsexuals. Journal of Voice. Septiembre de 2007;21(5):591-603. Doi: https://doi.org/10.1016/j.jvoice.2006.05.005

15. Gelfer MP, Tice RM. Perceptual and Acoustic Outcomes of Voice Therapy for Male-to-Female Transgender Individuals Immediately After Therapy and 15 Months Later. Journal of Voice. Mayo de 2013;27(3):335-47. Doi: https://doi.org/10.1016/j.jvoice.2012.07.009

16. Van Damme S, Cosyns M, Deman S, Van den Eede Z, Van Borsel J. The Effectiveness of Pitch-raising Surgery in Male-to-Female Transsexuals: A Systematic Review. Journal of Voice. Marzo de 2017;31(2):244.e1-244.e5. Doi: https://doi.org/10.1016/j.jvoice.2016.04.002

17. Remacle M, Matar N, Morsomme D, Veduyckt I, Lawson G. Glottoplasty for Male-to-Female Transsexualism: Voice Results. Journal of Voice. Enero de 2011;25(1):120-3. Doi: https://doi.org/10.1016/j.jvoice.2009.07.004

18. Mastronikolis NS, Remacle M, Biagini M, Kiagiadaki D, Lawson G. Wendler Glottoplasty: An Effective Pitch Raising Surgery in Male-to-Female Transsexuals. Journal of Voice. Julio de 2013;27(4):516-22. Doi: https://doi.org/10.1016/j.jvoice.2013.04.004

19. Meister J, Hagen R, Shehata-Dieler W, Kühn H, Kraus F, Kleinsasser N. Pitch Elevation in Male-to-female Transgender Persons - the Würzburg Approach. Journal of Voice. Marzo de 2017;31(2):244.e7-244.e15. Doi: https://doi.org/10.1016/j.jvoice.2016.07.018 
20. Anderson JA. Pitch Elevation in Trangendered Patients: Anterior Glottic Web Formation Assisted by Temporary Injection Augmentation. Journal of Voice. Noviembre de 2014;28(6):816-21. Doi: https://doi.org/10.1016/j.jvoice.2014.05.002

21. Orloff LA, Mann AP, Damrose JF, Goldman SN. Laser-Assisted Voice Adjustment (LAVA) in Transsexuals: The Laryngoscope. Abril de 2006;116(4):655-60. Doi: https:// doi.org/10.1097/01.mlg.0000205198.65797.59

22. Geneid A, Rihkanen H, Kinnari TJ. Long-term outcome of endoscopic shortening and stiffening of the vocal folds to raise the pitch. Eur Arch Otorhinolaryngol. Diciembre de 2015;272(12):3751-6. Doi: https://doi.org/10.1007/s00405-015-3721-7

23. Ganong LH. Integrative reviews of nursing research. Res Nurs Health. Febrero de 1987;10(1):1-11. Doi: https://doi.org/10.1002/nur.4770100103

24. Page MJ, McKenzie JE, Bossuyt PM, Boutron I, Hoffmann TC, Mulrow CD, et al. The PRISMA 2020 statement: An updated guideline for reporting systematic reviews. PLoS Med. 29 de marzo de 2021;18(3):e1003583. Doi: https://doi.org/10.1136/bmj.n71

25. Casado JC, O’Connor C, Angulo MS, Adrián JA. Wendler Glottoplasty and Voice-Therapy in Male-to-Female Transsexuals: Results in Pre and Post-Surgery Assessment. Acta Otorrinolaringologica (English Edition). Marzo de 2016;67(2):83-92. Doi: https://doi. org/10.1016/j.otoeng.2015.02.003

26. Thomas JP, MacMillan C. Feminization laryngoplasty: assessment of surgical pitch elevation. Eur Arch Otorhinolaryngol. Septiembre de 2013;270(10):2695-700. Doi: https:// doi.org/10.1007/s00405-013-2511-3

27. Dhejne C, Van Vlerken R, Heylens G, Arcelus J. Mental health and gender dysphoria: A review of the literature. International Review of Psychiatry. 2 de enero de 2016;28(1):4457. Doi: https://doi.org/10.3109/09540261.2015.1115753

28. Joseph A, Cliffe C, Hillyard M, Majeed A. Gender identity and the management of the transgender patient: a guide for non-specialists. J R Soc Med. Abril de 2017;110(4):14452. Doi: https://doi.org/10.1177/0141076817696054

29. Fernández Rodríguez M, Guerra Mora P, Martín Sánchez E, Grupo GIDSEEN. [Characteristics of Adolescents with Gender Dysphoria Referred to the Gender Identity Treatment Unit]. Rev Esp Salud Publica. 1 de febrero de 2017;91. Disponible en: https://tinyurl.com/yenmrbyq

30. Miguel T, Vierge SA, Schaffer I, Ansio FG, Montenegro JS, Antonio IED, et al. Una reflexión sobre el concepto de género alrededor de la transexualidad. Rev Asoc Esp Neuropsiq. 2008;28(1):201-26. Disponible en: https://n9.cl/9b8qv 